\title{
Statistical phase propagation and dispersion analysis of low frequency waves in the magnetosheath
}

\author{
S. Schäfer ${ }^{1}$, K.-H. Glassmeier ${ }^{1}$, Y. Narita ${ }^{1}$, K. H. Fornaçon ${ }^{1}$, I. Dandouras ${ }^{2}$, and M. Fränz ${ }^{3}$ \\ ${ }^{1}$ Institut für Geophysik und extraterrestrische Physik, TU Braunschweig, Germany \\ ${ }^{2}$ Centre d'Etude Spatiale des Rayonnements, Toulouse, France \\ ${ }^{3}$ Max-Planck-Institut für Sonnensystemforschung, Katlenburg-Lindau, Germany
}

Received: 4 October 2004 - Revised: 17 June 2005 - Accepted: 30 June 2005 - Published: 30 November 2005

\begin{abstract}
We present the results of a statistical analysis of low-frequency fluctuations in the high latitude regions of the dayside magnetosheath using CLUSTER as a wave telescope. Magnetic field observations are used to determine wave propagation directions and wave numbers for selected frequencies. Using observations of the plasma flow velocity we correct for the Doppler shift, in order to calculate frequencies and phase velocities in the plasma rest frame. This provides us with the possibility to perform a statistical dispersion analysis and to investigate various wave properties, such as the phase velocity and the propagation angle between $\boldsymbol{k}$ and $\boldsymbol{B}$. The analysis of dispersion distributions and Friedrichs diagrams results in the identification of different wave populations. We find a multiplicity of standing structures (mirror modes) convected with the plasma flow and a large number of Alfvénic waves. The results confirm previous magnetosheath wave studies, such as ISSE or AMPTE spacecraft observations, but we also find a small number of mirror mode-like waves that have propagation speeds up to the local Alfvén velocity, quasi-perpendicular to the magnetic field.
\end{abstract}

Keywords. Magnetospheric physics (magnetosheath; plasma waves and instabilities) - Space plasma physics (shock waves)

\section{Introduction}

The terrestrial magnetosheath constitutes the interface between the interplanetary medium and the magnetosphere of the Earth. Low-frequency fluctuations are dominating the magnetosheath plasma driven by solar wind dynamic pressure variations, plasma instabilities and other effects. It is generally accepted, that in the linearized Vlasov theory, Alfvén-proton-cyclotron mode and mirror mode are the predominant low-frequency wave modes in the magnetosheath

Correspondence to: S. Schäfer

(seb.schaefer@tu-bs.de)
(Schwartz et al., 1996), whereas the nonlinear treatment and fluid picture can lead to a variety of slow and Alfvén modes (Stasiewicz, 2005). Alfvén-proton-cyclotron waves are lefthand polarized and propagate preferably parallel to the magnetic field direction. In contrast to that, mirror modes are linear polarized and non-propagating waves, or standing structures, in the plasma rest frame. The growth of these modes is mainly affected by the temperature anisotropy $T_{\perp, p}>T_{\|, p}$ and different values of the plasma $\beta$ (Denton, 2000), where $T_{\perp, p}$ and $T_{\|, p}$ are the proton temperatures perpendicular and parallel to the background magnetic field $\boldsymbol{B}$. Several reasons are known that cause the onset of temperature anisotropies, for example, ion beams which are reflected at the quasiperpendicular bow shock and move downstream into the magnetosheath afterwards because of the gyration around the magnetic field (Sckopke et al., 1990). Also, the extension and compression of the magnetic field adjacent to the magnetosphere can lead to temperature anisotropies (Crooker and Siscoe, 1977).

Both the non-compressional Alfvénic and the compressional mirror mode have been observed in the magnetosheath. Hubert et al. (1998) used magnetic field measurements of the ISEE satellites and suggested different regions of preferred wave modes within the magnetosheath. Near the magnetopause they detected mirror modes exclusively, but in the vicinity of the bow shock, only Alfvén modes were observed, while Lacombe et al. (1992) detected Alfvén and mirror modes in this region. In the middle of the magnetosheath Hubert et al. (1998) and Anderson et al. (1994) identified both wave modes. Using measurements of the AMPTE satellites further observations of standing waves in the inner magnetosheath are described by, for example, Balikhin et al. (2001) and Chisham et al. (1999), whereas Denton et al. (1995) detected mirror modes near the magnetopause.

A major unresolved problem in previous analysis of the plasma wave properties within the magnetosheath is the identification of the wave vector $\boldsymbol{k}$. Using single or double spacecraft measurements traditional analysis tools allow one only to estimate $\boldsymbol{k}$ under limited circumstances. For example, 


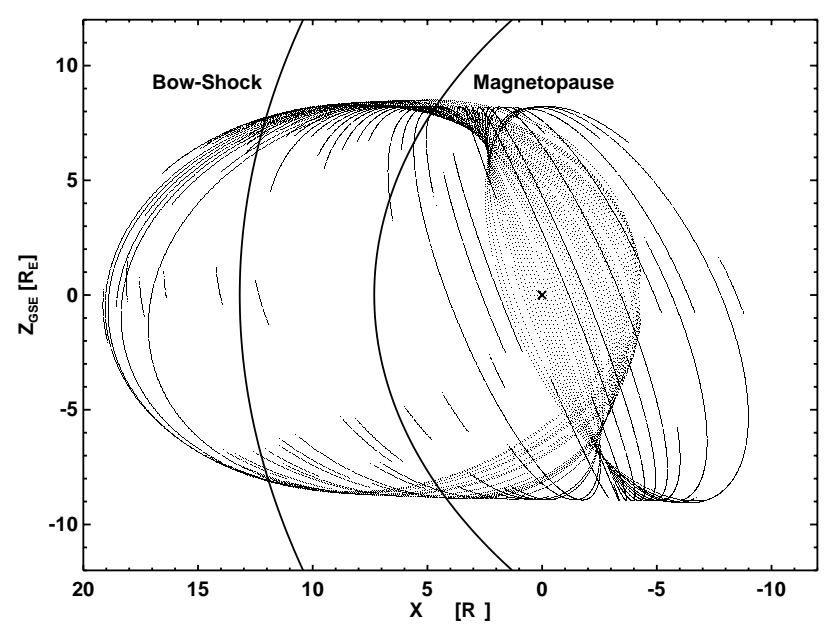

Fig. 1. Positions $\boldsymbol{r}_{B}$ of the spacecraft between February and June 2002 displayed in the $\left(X_{G S E}-Z_{G S E}\right)$-plane.

the direction of the wave vector can be determined by using the minimum variance analysis (MVA), but, in general, this direction has an ambiguity of $180^{\circ}$ and the wave number $|\boldsymbol{k}|$ cannot be determined uniquely. Moreover, the MVA is not applicable to linear polarized waves, such as mirror modes, so that further assumptions are necessary to estimate $\boldsymbol{k}$ (Chisham et al., 1999). A much more reliable technique to determine $\boldsymbol{k}$ was developed by, for example, Neubauer and Glassmeier (1990), Pinçon and Lefeuvre (1991) and Motschmann et al. $(1995,1996)$. This method is called wave telescope or k-filtering and gives one the possibility to determine both the direction and wave number in the spacecraft frame using only the magnetic field measurements. We compare the results of the MVA technique and the wave telescope in Sect. 4.

The wave telescope analysis uses simultaneous measurements of the magnetic field vector taken from at least four different points in space and allows one to determine $\boldsymbol{k}$ for any given wave frequency $\omega$. The wave telescope analysis was successfully applied to measurements by Glassmeier et al. (2001) and Narita et al. (2004) using magnetic field measurements of the fluxgate magnetometers on board the four CLUSTER satellites (Balogh et al., 2001). The wave telescope technique is in essence a generalized minimum variance technique which allows to determine the power density $P\left(\omega, \boldsymbol{k}, \boldsymbol{r}_{B}\right)$, where $\boldsymbol{r}_{B}=\frac{1}{4} \sum_{i=1}^{4} \boldsymbol{r}_{i}$ is the barycenter of the four CLUSTER satellites. Due to the finite distance between the spacecraft, aliasing effects need to be taken into account and $\boldsymbol{k}$ can only be determined within a Nyquistsphere whose radius depends on the spacecraft separation (Neubauer and Glassmeier, 1990; Glassmeier et al., 2001).

The fluctuations of interest in this study are embedded in the magnetosheath flow. As we are interested in the physical properties of these fluctuations in the plasma rest frame, we need to correct for the Doppler effect. This requires a detailed knowledge of the plasma flow velocity $\boldsymbol{V}_{S W}$ and $\boldsymbol{k}$, as measured in the spacecraft frame. If this information is available, the rest frame angular frequency $\omega_{\text {rest }}$ is given as

$\omega_{\text {rest }}=\omega_{s c}-\boldsymbol{k} \cdot \boldsymbol{V}_{S W}$,

where $\omega_{s c}$ denotes the angular frequency in the spacecraft frame. The velocity of the satellites is small $\left(V_{s c} \approx 2 \mathrm{~km} / \mathrm{s}\right)$ compared to the plasma flow velocity $\left(V_{S W}>100 \mathrm{~km} / \mathrm{s}\right)$. Thus, we neglect $V_{s c}$ when calculating the Doppler shift. To determine the plasma flow velocity we use the measurements of the Cluster Ion Spectrometry (CIS) instrument on board CLUSTER (Rème et al., 2001), data of which are also used to determine the ion density and the Alfvén velocity.

For selected time intervals and regions specified below we determine $P\left(\omega, \boldsymbol{k}, \boldsymbol{r}_{B}\right)$, as well as frequencies and phase velocities in the plasma rest frame. The analysis is restricted to the frequency range $\omega_{s c}=(0.063,1.571) \mathrm{Hz}$. For this frequency range we are able to provide in depth information about key wave propagation parameters in the terrestrial magnetosheath, as well as a study of the statistical dispersion properties of these waves.

\section{Data selection}

A primary requirement for selecting suitable time intervals is that the satellite configuration should be close to an ideal tetrahedron with small distances of about $100 \mathrm{~km}$ to avoid spatial aliasing and to ensure the identification of a large range of wave numbers. This requirement is satisfied in the time period from February 2002 to June 2002, where the available configurations are sensitive to wave numbers up to $k=0.032 \mathrm{~km}^{-1}$ and wavelengths above $\lambda=200 \mathrm{~km}$. The average spatial distribution of the spacecraft position $\boldsymbol{r}_{B}$ in the $\left(X_{G S E}-Z_{G S E}\right)$ plane reveals that the satellites are mainly located in both high latitude $\left(X_{G S E}-Y_{G S E}\right)$ planes at $Z_{G S E}>6 R_{E}$ and $Z_{G S E}<6 R_{E}$ (Fig. 1). Thus, we consider only time intervals in these planes to ensure study of magnetosheath wave properties in a wide area.

As we study the wave properties in the plasma rest frame, information about the plasma flow velocity of the given time interval is required. If there are no measurements of $V_{S W}$, we are not able to determine the Doppler shift. We furthermore restrict our analysis to events containing fluctuations with typical amplitudes, in order to consider only wave activities that usually exist in the magnetosheath. We define a magnetic field variance measure $\sigma$ via

$\sigma^{2}=\frac{\left\langle|\boldsymbol{B}|^{2}\right\rangle-|\langle\boldsymbol{B}\rangle|^{2}}{\left\langle|\boldsymbol{B}|^{2}\right\rangle}$,

i.e. we determine the average deviation of the magnetic field strength compared to the mean, background magnetic field strength normalized to its magnitude $|\boldsymbol{B}|$. We determine $\sigma^{2}$ for the available magnetosheath data by averaging over 256$\mathrm{s}$ intervals. This leads to a mean value for the variance of $\sigma^{2} \approx 0.15$ for the magnetosheath. Thus, we select intervals with fluctuations that have values of $\sigma^{2}$ between 0.05 and 0.4 . 


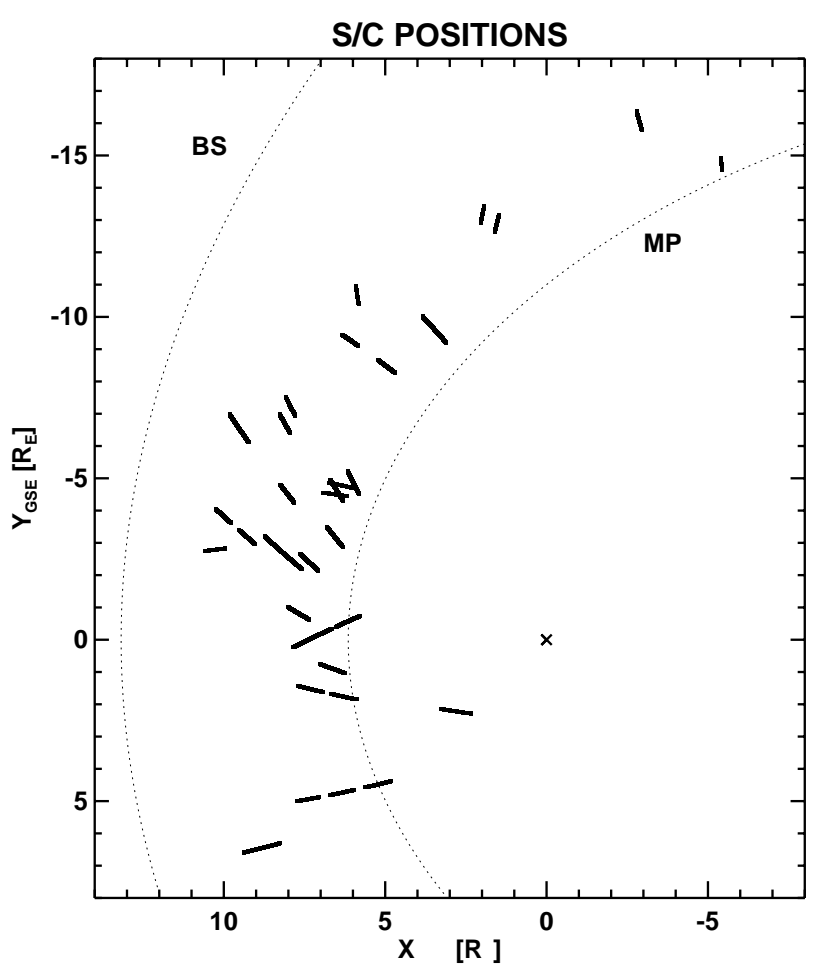

Fig. 2. Positions $\boldsymbol{r}_{B}$ of the selected time intervals displayed in the $\left(X_{G S E}, Y_{G S E}\right)$-plane. The dotted lines mark an estimated location of the magnetopause and bow shock.

The criteria described above result in a selection of 37 time intervals that are located in high-latitude planes at $\left|Z_{G S E}\right|>6 R_{E}$. In these planes the intervals cover the dayside magnetosheath from 05:00 to 15:00 in local time (Fig. 2). In addition, the intervals are located rather adjacent to the magnetopause than to the bow shock. For interpreting the results of the statistical analysis we have to consider the spatial distribution of the time intervals, because the origin of plasma waves depends on the region of the magnetosheath.

Wave properties will also depend on the plasma parameter $\beta$, as well as the proton temperature anisotropy given by $A=T_{\perp} / T_{\|}-1$. Using plasma measurements from the CIS experiment (Rème et al., 2001) we find that for the time intervals analyzed, $\beta$ varies between 1.3 and 21.8 while the anisotropy $A$ covers the range -0.17 to 0.91 .

\section{Analysis procedure}

We explain the procedure of the wave telescope analysis by considering as an example the time interval 18 February 2002, 05:50-06:24 UT. The barycenter of the CLUSTER satellites is located at $\boldsymbol{r}_{B}=(4.83,4.39,8.53) R_{E}$ in the highlatitude afternoon section of the magnetosheath. The spacecraft are spaced at distances of about $100 \mathrm{~km}$ and form an almost ideal tetrahedron. This means that we find ideal conditions for the waves telescope analysis which allow us to

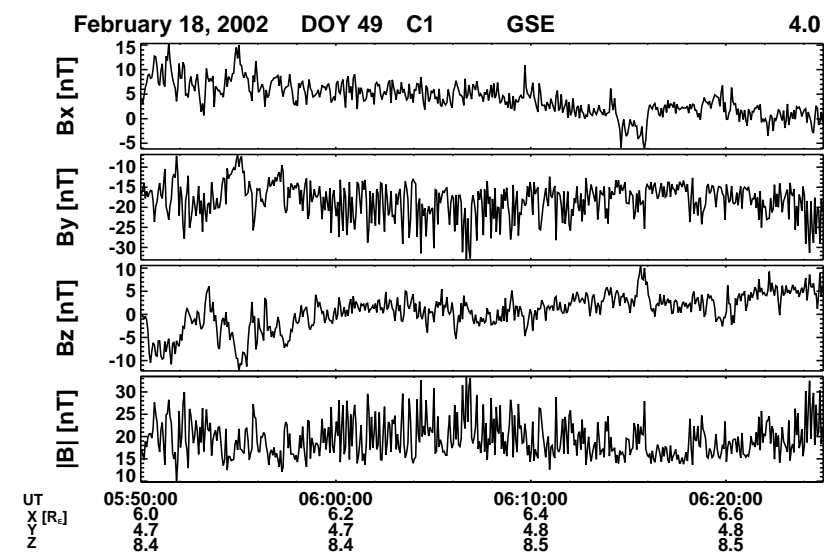

Fig. 3. Measurements of the magnetic field vector components and the magnitude $B$ made by spacecraft $C 1$ on 18 February 2002, 05:50-06:24.

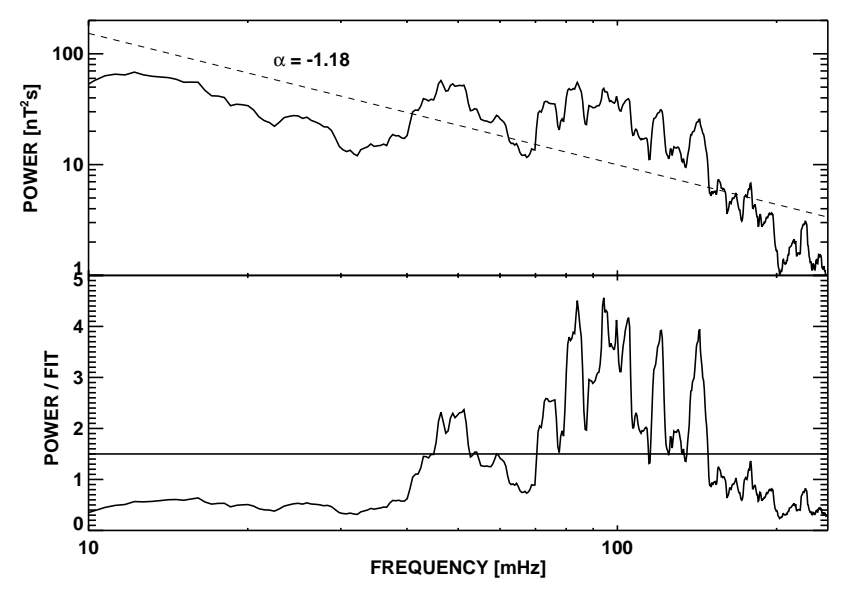

Fig. 4. Upper panel: Frequency power spectrum of the interval from 05:50 to 06:24 on 18 February 2002. The dashed line represents the linear fit of the spectrum. The lower panel displays the ratio of the power spectrum and the linear fit.

determine wave numbers up to $k_{N y}=0.0314 \mathrm{~km}^{-1}$ and wavelengths above $200 \mathrm{~km}$.

Figure 3 displays the magnetic field vector components $B_{x}, B_{y}, B_{z}$ and the magnitude $|\boldsymbol{B}|$. The mean magnetic field vector is $\langle\boldsymbol{B}\rangle=(-4.3,-18.1,-0.73) \mathrm{nT}$ and the mean magnitude is $|\langle B\rangle|=18.6 \mathrm{nT}$ in the analyzed time interval. The CIS instrument on board the satellite $\mathrm{C} 1$ measures a mean plasma flow velocity vector $\left\langle\boldsymbol{V}_{S W}\right\rangle=(-179,63,114) \mathrm{km} / \mathrm{s}$, with $\left|\left\langle\boldsymbol{V}_{S W}\right\rangle\right|=222 \mathrm{~km} / \mathrm{s}$, and a mean ion number density $\langle\rho\rangle=40 \mathrm{~cm}^{-3}$.

The frequency power spectrum of the example interval is shown in Fig. 4 (upper panel). It contains a multiplicity of spectral peaks, to which we restrict our analysis. For an unambiguous determination of these power spectral maxima we define as peak frequencies all those frequencies where the spectral power level is larger than 1.5 times the trend power spectral value. The trend power spectrum is determined by modelling the observed power spectrum in the frequency 


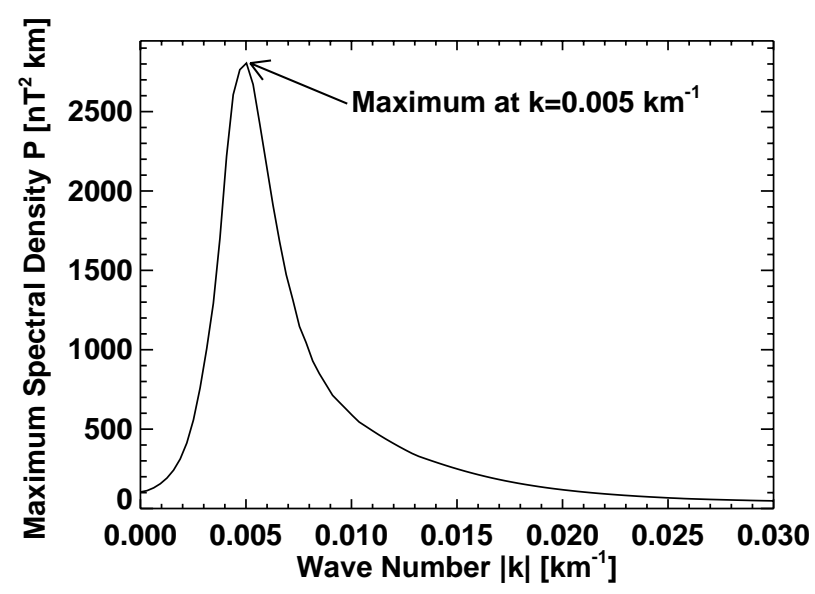

Fig. 5. Spatial wave spectrum at $f=0.133 \mathrm{~Hz}$.

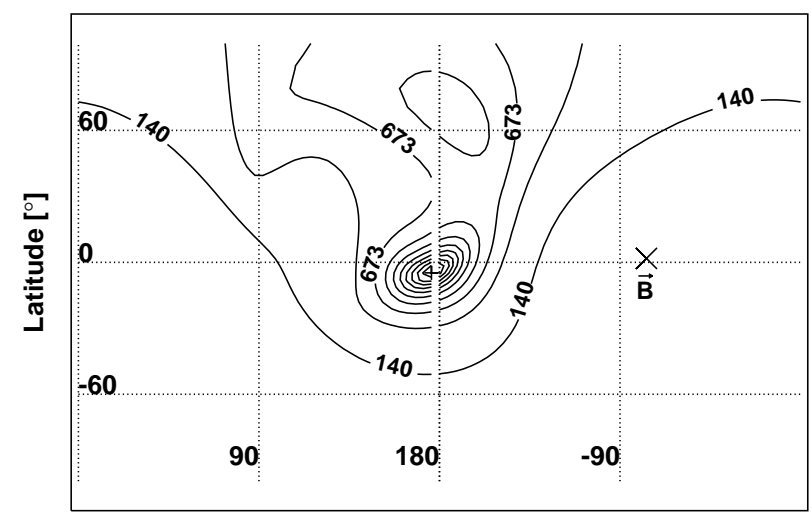

Longitude $\left[^{\circ}\right]$

Fig. 6. Spatial power distribution of $f=0.133 \mathrm{~Hz}$ and $k=0.005 \mathrm{~km}^{-1}$. The cross denotes the direction of the ambient magnetic field $\boldsymbol{B}$.

range $(0.01,0.25) \mathrm{Hz}$ as a power law spectrum $P(f) \propto f^{\alpha}$. In this way we determine the peak frequencies for any selected time interval. For these peak frequencies we then calculate the corresponding wave vectors, as described by Glassmeier et al. (2001) or Narita et al. (2004). In the present case seven different frequencies have been determined: $f_{s c}=0.051$, $0.06,0.104,0.121,0.130,0.133$ and $0.143 \mathrm{~Hz}$. Here, the subscript sc indicates frequencies as measured in the spacecraft frame of reference. The wave telescope technique is applied to the frequency range $\Delta f=f_{s c} \pm 0.002 \mathrm{~Hz}$ to ensure sufficient statistical significance of our results.

Results of the wave telescope analysis are displayed in Figs. 5 and 6 for $f_{s c}=0.133 \mathrm{~Hz}$. In the present case the shell-max spatial wave spectrum is displayed, i.e. the maximum spectral density value on each wave number shell is represented (e.g. Glassmeier et al., 2001). The spectrum contains a clear maximum at the wave number $|\boldsymbol{k}|=0.005 \mathrm{~km}^{-1}$. For the given wave number the directional power spectrum is displayed in Fig. 6. Latitude and longitude in GSE coordinates are used, i.e. $\left(\phi_{\text {lon }}=0^{\circ}, \phi_{\text {lat }}=0^{\circ}\right)$ corresponds to the direction of $X_{G S E}$ and $\left(\phi_{l o n}= \pm 180^{\circ}, \phi_{\text {lat }}=0^{\circ}\right)$ is parallel to $-X_{G S E}$. The $\pm Y_{G S E}$ directions are represented by $\left(\phi_{\text {lon }}= \pm 90^{\circ}, \phi_{\text {lat }}=0^{\circ}\right)$ and the $\pm Z_{G S E}$ directions by $\left(\phi_{\text {lon }}=0^{\circ}, \phi_{\text {lat }}= \pm 90^{\circ}\right)$. The position of maximum power is interpreted as the direction of the wave vector $\boldsymbol{k}$.

A wave event is identified if there is a directional region where the power density is above $70 \%$ of the average wave power value of any wave number shell. In the present case, a wave event can be identified with the wave propagating in the direction $\phi_{\text {lat }}=-6^{\circ}$ and $\phi_{\text {lon }}=176^{\circ}$ which corresponds to a propagation almost parallel to $X_{G S E}$. Our analysis thus allows one to identify a wave with $\boldsymbol{k}=(-4.99,0.35,-0.52) \times 10^{-3} \mathrm{~km}^{-1}$ in the time interval 05:50 to $06: 24$ on 18 February 2002.

This corresponds to a phase velocity in the spacecraft frame $V_{P h, s c}=\omega_{s c} /|\boldsymbol{k}|=166 \mathrm{~km} / \mathrm{s}$, where $\omega_{s c}=2 \pi \times 0.133 \mathrm{~Hz}$. Taking into account the Doppler effect we obtain a negative frequency $\omega_{\text {rest }}=-0.0206 \mathrm{~Hz}$, that leads to a negative value of the phase velocity $V_{P h \text {,rest }}=\omega_{\text {rest }} /|\boldsymbol{k}|=-4.09 \mathrm{~km} / \mathrm{s}$ in the plasma rest frame. The Doppler shift is larger than the detected frequency $\omega_{s c}$. If we define the direction of the phase velocity vector $V_{P h, \text { rest }}$ to be parallel to the propagation direction given by the wave vector $\boldsymbol{k}$, one needs to change the sign of $\boldsymbol{k}$ in those cases where $\omega_{\text {rest }}$ is negative. In these special circumstances, fluctuations convected with the plasma flow in the $-X_{G S E}$ direction towards the magnetopause propagate with a small phase velocity parallel to $X_{G S E}$ towards the bow shock. The wave event identified in the interval $05: 50$ to $06: 24$ on 18 February 2002 is such a special case, where the fluctuations also propagate roughly perpendicular to the background magnetic field; the angle between $\boldsymbol{k}$ and $\boldsymbol{B}$ is $\theta_{k B}=107^{\circ}$.

The interval on 18 February 2002 was recently investigated by Sahraoui et al. (2003, 2004). They compared $k$-filtering analysis from the FGM and STAFF (SpatioTemporal Analysis of Field Fluctuations) instruments and obtained consistent results. They concluded, that waves around 05:30 UT were mirror modes. This is in accord with the results of our analysis, since the fluctuation exhibits a negligible phase velocity and a quasi-perpendicular propagation angle.

To determine the accuracy of our analysis procedure we estimate the relative error $\Delta V_{P h \text {,rest }} / V_{P h \text {,rest }}$ The errors of $\omega_{s c}, k$, and $V_{S W}$ enter the determination of $\omega_{\text {rest }}$ via Eq. (1) and $V_{P h \text {,rest }}=\omega_{\text {rest }} / k$. We assume an error $\Delta \omega_{s c}=0.013 \mathrm{~Hz}$ based on the frequency analysis interval used for the wave telescope technique. The accuracy of the wave vector is of the order of between $\Delta k=0.00025 \mathrm{~km}$. For the plasma flow velocity a relative error of $\Delta V_{S W} / V_{S W} \leqq 0.07$ is used. The calculation of errors for the given phase velocity leads to $V_{P h \text {,rest }}=(-4.09 \pm 15.9) \mathrm{km} / \mathrm{s}$. The relative error is $\Delta V_{p h} / V_{p h}=3.9$. As mentioned before, the sign of the phase velocity is important to identify the propagation direction. In this case the error $\Delta V_{p h}$ exceeds the phase velocity $V_{p h}$, so that the propagation direction is ambiguous. 


\section{Results of the statistical analysis}

We apply the analysis method described in the previous section to the selected 37 time intervals within the dayside magnetosheath, resulting in a statistical survey of wave properties in this region. All in all, we have analyzed 407 frequencies and have identified 516 wave vectors, i.e. in some cases we find more than one wave vector at a given frequency. In the following we denote a frequency and its corresponding wave vector $(\omega, \boldsymbol{k})$ as wave event.

The $(\omega, k)$-distributions found are displayed in Fig. 7 for both frequencies in the spacecraft, as well as in the plasma rest frame. Frequencies are normalized to the protoncyclotron frequency $\Omega_{c}=e|\boldsymbol{B}| / m_{p}$ and wave numbers to $\Omega_{c} / V_{A}$, where $V_{A}^{2}=B^{2} /\left(2 \mu_{0} \rho m_{p}\right)$ is the Alfvén velocity, $e$ the electron charge, $m_{p}$ the proton mass and $\mu_{0}$ the magnetic susceptibility. Frequencies for which wave events have been detected are almost regularly distributed up to a normalized frequency of 0.95 in the spacecraft frame and 0.4 in the plasma rest frame. The associated normalized wave numbers range up to 0.9 , with the majority of events exhibiting wave numbers up to 0.5 . The range of phase velocities in the rest frame is also rather broad, i.e. various phase velocities can be identified.

In the $\left(\omega_{\text {rest }}, \mathrm{k}\right)$-distribution we can distinguish at least three different populations of wave events. The first is in the frequency range $\left(-0.05 \Omega_{c}, 0.05 \Omega_{c}\right)$, centered around $\omega_{\text {rest }} \simeq 0 \mathrm{~Hz} ; 351$ events belong to this population. Their normalized wave numbers are in the range $(0.0,0.4)$. We interpret this group of wave events as spatial structures at rest in the plasma frame and convected past the spacecraft. The second population, containing 153 events, exhibits normalized frequencies in the range $(0.05,0.4)$ and the corresponding waves propagate in the same direction as in the spacecraft frame. For the remaining 12 events, the third group, we find negative plasma rest frame frequencies lower than $-0.05 \Omega_{c}$. These structures propagate from the magnetopause to the bow shock.

In the following we shall discuss the different wave events found according to their wave phase velocity, their propagation direction by using a Friedrichs-diagram representation, their wave numbers and wave lengths as well as their propagation direction with respect to the GSE coordinate system. Figure 8 displays a histogram of wave phase velocities $V_{P h, s c}=\omega_{s c} / k$ and $V_{P h \text {,rest }}=\omega_{\text {rest }} / k$, normalized to the Alfvén velocity of the particular analysis interval. Averaged over all analysis intervals, the Alfvén velocity is $V_{A} \approx 100 \mathrm{~km} / \mathrm{s}$, while the average magnetosheath flow velocity is $V_{S W} \approx 200 \mathrm{~km} / \mathrm{s}$. In the spacecraft frame of reference the phase velocities exhibit a broad, almost symmetric distribution centered at a normalized velocity of about 1.5 . In the plasma frame of reference, however, two different populations emerge. For 374 wave events the normalized velocity is smaller than 0.3. We interpret these events as standing or slowly propagating structures convected with the magnetosheath flow. For the remaining 142 wave events we find

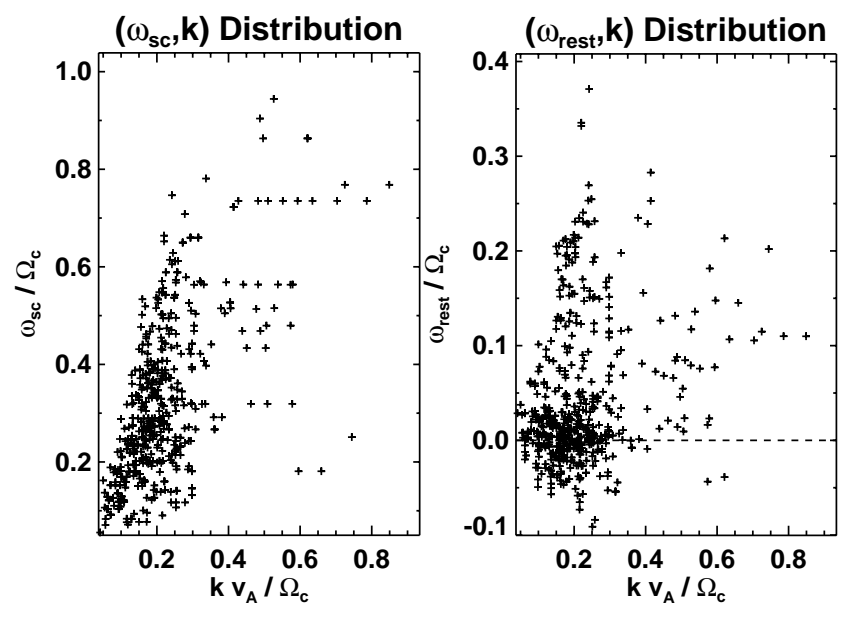

Fig. 7. The $(\omega, k)$-distributions in the spacecraft frame (left) and in the plasma rest frame (right). $\Omega_{c}$ is the proton cyclotron frequency and $V_{A}$ the Alfvén velocity.

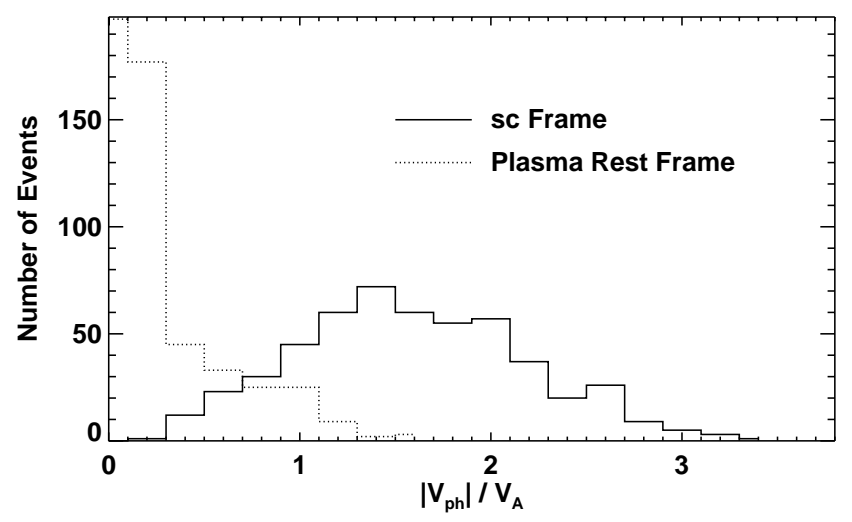

Fig. 8. Histogram of phase velocities in the spacecraft frame and in plasma rest frame, taking into account the Doppler shift.

$V_{P h \text {,rest }}>0.3 V_{A}$; this means that these waves are propagating waves in the plasma rest frame.

Figure 9 displays the distribution of the small phase velocity $\left(\left|V_{P h, \text { rest }}\right|<0.3 V_{A}\right)$ population with phase velocities in the interval $(-0.3,0.3)$. To decide whether these waves are indeed propagating in the plasma rest frame or are just standing structures, a detailed error analysis for the phase velocities has been performed. For the majority of the events (solid line in Fig. 9), 265 out of 374, the error estimation gives a relative error $\Delta \omega_{\text {rest }} / \omega_{\text {rest }}>1$. This implies that the sign of the rest frame velocity $V_{P h \text {,rest }}$ is undetermined. Accordingly, the propagation directions are ambiguous; therefore, no clear propagation behavior can be determined. We classify these wave events with small phase velocities as standing structures. However, for 81 wave events with positive and 28 wave events with negative $V_{P h \text {,rest }}$, we determine a relative error $\Delta V_{P h \text {,rest }} / V_{P h \text {,rest }}<1$ (dotted line Fig. 9). For these events the propagation direction is known unambiguously, and the events can be identified as slow propagating 


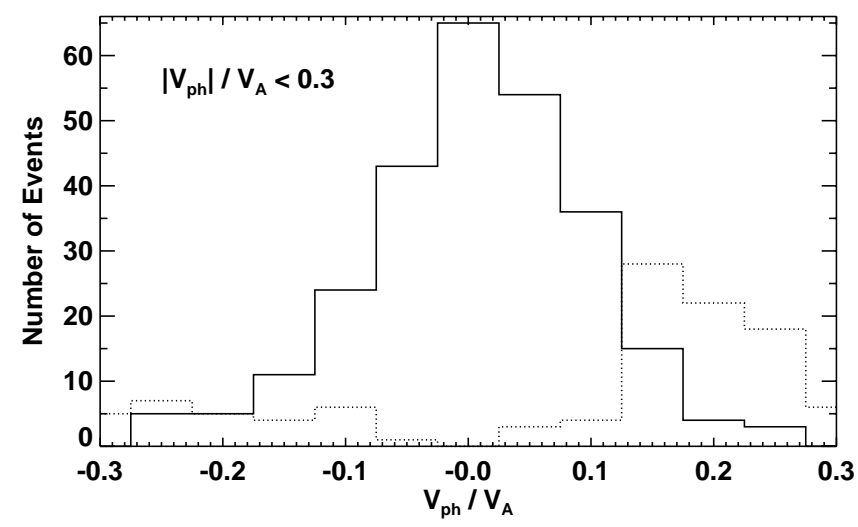

Fig. 9. Histogram of slow phase velocities: standing structures (solid) and slow propagating waves (dotted) in the plasma rest frame.

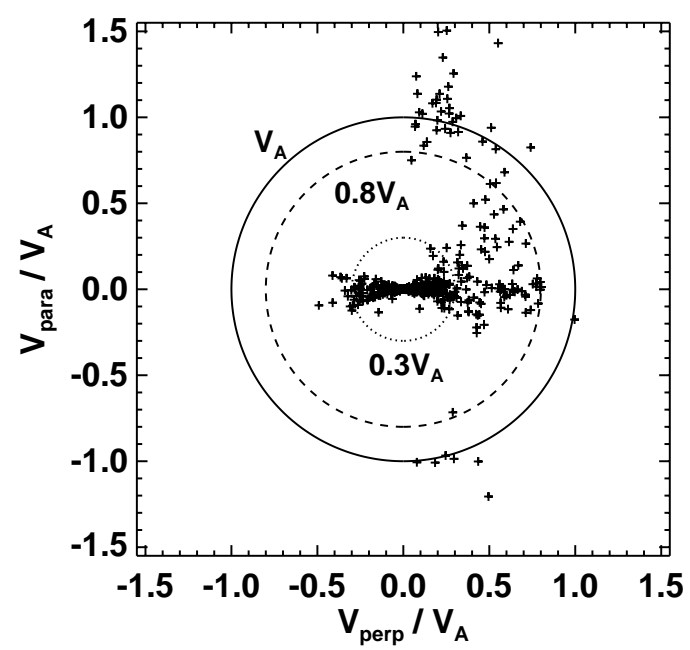

Fig. 10. Friedrichs diagram of magnetosheath wave events. The radii of the circles denote the phase velocities $0.3 V_{A}$ (dotted), $0.8 V_{A}$ (dashed) and $V_{A}$ (solid).

waves. Concerning the 142 wave events with fast phase velocities $V_{P h \text {,rest }}>0.3 V_{A}$, almost all of them exhibit relative errors $\Delta V_{P h \text {,rest }} / V_{P h \text {,rest }}<1$, which implies that their propagation direction is also properly determined.

To further analyze the wave events found, we determine the angle $\theta_{k B}$ between the background magnetic field $\boldsymbol{B}$, averaged over the respective analysis interval, and the wave vector $\boldsymbol{k}$, which is parallel to the phase velocity vector $\boldsymbol{V}_{\boldsymbol{p h}}$, according to our definition. This allows one to display wave phase velocities using a polar plot with respect to the direction of the background magnetic field, which is comparable to the Friedrichs diagram, of MHD waves. Figure 10 shows such an experimental Friedrichs-diagram using all wave events found. $V_{\text {para }}$ and $V_{\text {perp }}$ denote the components of the phase velocity vector parallel and perpendicular to the ambient magnetic field $\boldsymbol{B}$. Negative values of $V_{\text {perp }}$ occur, if wave vectors change their sign, so that the Doppler shift leads to negative frequencies (see Sect. 3). Negative values

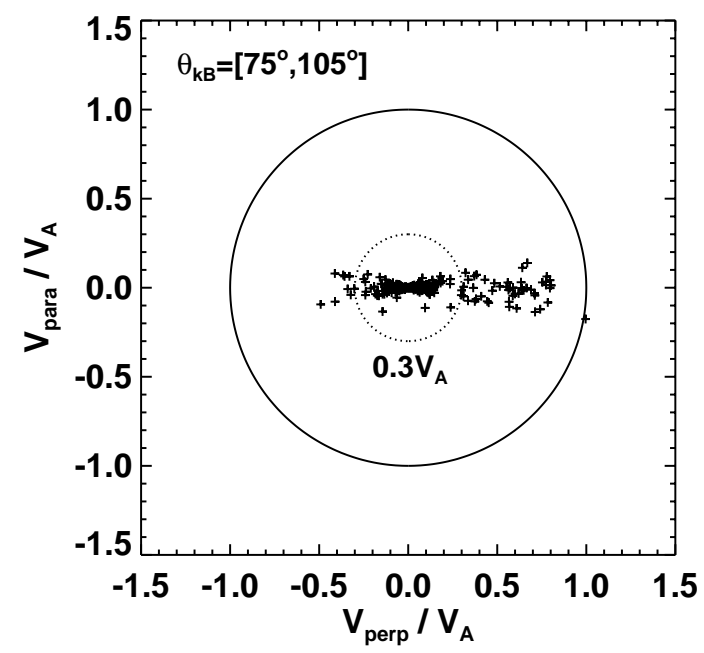

Fig. 11. Friedrichs diagram of perpendicular propagating wave events that are identified as standing structures $\left(V_{p h}<0.3 V_{A}\right)$ and perpendicular propagating fluctuations $\left(V_{p h}>0.3 V_{A}\right)$.

of $V_{\text {para }}$ are due to wave vectors directed antiparallel to the magnetic field. To simplify matters we combine all wave events to a single Friedrichs diagram, although the plasma properties differ for each time interval; only the Alfvén velocity is used here as a normalization parameter.

As before, different wave populations can be distinguished. This time the propagation direction is used as a classification tool. Defining all waves with propagation directions in the range $\theta_{k B}=\left(75^{\circ}, 105^{\circ}\right)$ as quasi-perpendicular propagating waves, allows one to identify 324 out of the 516 events to belong to this class. Their Friedrichs diagram is displayed in Fig. 11. The majority of them, 265 events, have small phase velocities, lower than $0.3 V_{A}$. This low velocity wave population is composed of standing structures and slow propagating waves already discussed and displayed before in Fig. 9. Theoretical investigations on waves in an anisotropic plasma indicate that wave structures with vanishing phase velocity and $\boldsymbol{k}$ perpendicular to $\boldsymbol{B}$ may be identified as mirror modes. Therefore, we assume, that the standing structures' population indicates the existence of mirror modes, as found in the magnetosheath in many previous investigations.

The remaining 59 quasi-perpendicular wave events $\left(\theta_{k B}=\left(75^{\circ}, 105^{\circ}\right)\right)$ are characterized by phase velocities above $0.3 V_{A}$, but smaller than the local Alfvén velocity. Mode identification of these events is more difficult. They cannot be interpreted as, for example, perpendicular propagating magnetoacoustic waves in a high $\beta$ plasma, as in this case their phase velocity should be larger than $V_{A}$. Kinetic effects cannot explain their small phase velocity, either. Krauss-Varban et al. (1994) have calculated phase velocities of kinetic plasma waves as a function of $\beta$ and $\theta$ for $\mathrm{k}$-values discussed here. They found that only the slow magnetosonic wave has phase velocities below $V_{A}$, which, for large values of $\theta$, become almost zero, also in a high $\beta$ plasma regime. To further identify these events, we have performed a cross- 
spectral analysis between plasma density oscillations and magnetic field magnitude variations, which indicates a predominantly out-of-phase relation between these two plasma parameters. This supports the idea of this population also representing mirror mode structures, but propagating ones. As a local instability analysis demonstrates that mirror modes are zero-frequency and non-propagating structures, we speculate these wave events are mirror modes modified so as to have finite frequencies. The growth of such mirror modes is explainable by several theories: In an inhomogeneous plasma the gradients of the plasma density or the magnetic field can lead to drift mirror modes with real finite frequencies (Hasegawa, 1969; Pokhotelov et al., 2001), as well as an asymmetric velocity distribution function (Gedalin et al., 2002). Johnson and Cheng (1997) suggest a global instability analysis of mirror modes, including the effects of gradients and plasma flow, resulting in global mirror modes with nonzero frequencies and phase velocities. These interpretations are also supported by the fact that about $50 \%$ of such mirror mode structures identified come from a single time interval located in the middle of the magnetosheath. A global instability analysis is thus required to unravel the properties of drift mirror or global mirror modes. However, such an analysis is beyond the scope of this statistical study. In the nonlinear wave theory the perpendicular propagating events can also be interpreted as slow mode solitones that have phase velocities smaller than $V_{P h}=V_{A}$ (Stasiewicz, 2004a,b, 2005).

The Friedrichs diagram of the remaining population of quasi-parallel $\left(\theta_{k B}=\left(0^{\circ}, 30^{\circ}\right)\right)$ and oblique $\left(\theta_{k B}=\left(30^{\circ}, 75^{\circ}\right)\right)$ propagating waves is shown in Fig. 12; 192 events belong to this population, which also contains as a subset the slow propagating waves with an unambiguous propagation direction discussed earlier. They are located in the Friedrichs-diagram around the lemniscate $V_{p h}=V_{A} \times \cos \theta$ (dotted line), i.e. the lemniscate of the Alfvén mode in the MHD-theory. Gary (1992), for example, determined the Friedrichs diagrams for kinetic wave modes under magnetosheath plasma conditions and found that the branch of the Alfvénic waves is comparable to that branch with the Alfvén phase velocity $V_{p h}=V_{A} \times \cos \theta$ of MHD-theory. It is thus tempting to identify the wave events displayed in Fig. 12 as Alfvénic wave structures in the magnetosheath. However, one has to check for the possibility of these events being slow mode waves. If the thermal pressure $p_{t h}$ becomes larger than the magnetic pressure $p_{B}=B^{2} / 2 \mu_{0}$, the sound velocity $C_{S}$ is higher than the Alfvén velocity $V_{A}$. Under the high $\beta=p_{t h} / p_{B}$ conditions observed in the magnetosheath, the phase velocity of the slow mode $V_{\text {slow }}^{2}=\frac{1}{2}\left(V_{A}^{2}+C_{S}^{2}-\sqrt{\left(V_{A}^{2}+C_{S}^{2}\right)^{2}-4 V_{A}^{2} C_{S}^{2} \cos ^{2} \theta}\right)$ is comparable to the phase velocity of Alfvén waves. Using the temperature measurement of the CIS instrument on board CLUSTER 1; we calculate the sound velocity $C_{S}=\sqrt{\gamma k_{B} T / m_{p}}$, where $m_{p}$ stands for the proton mass and $\gamma$ for the polytropic index. The condition $C_{S}>V_{A}$ is satisfied for all analyzed time intervals; thus, the wave events of the Alfvénic wave population can be interpreted as slow modes, as well as

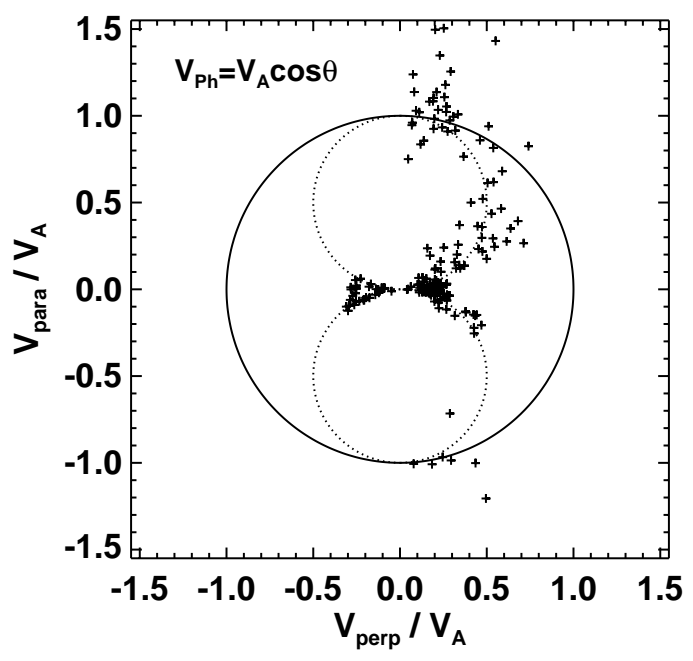

Fig. 12. Friedrichs diagram of Alfvén-like wave events featuring phase velocities comparable to $V_{A} \times \cos \theta$ (dotted line).

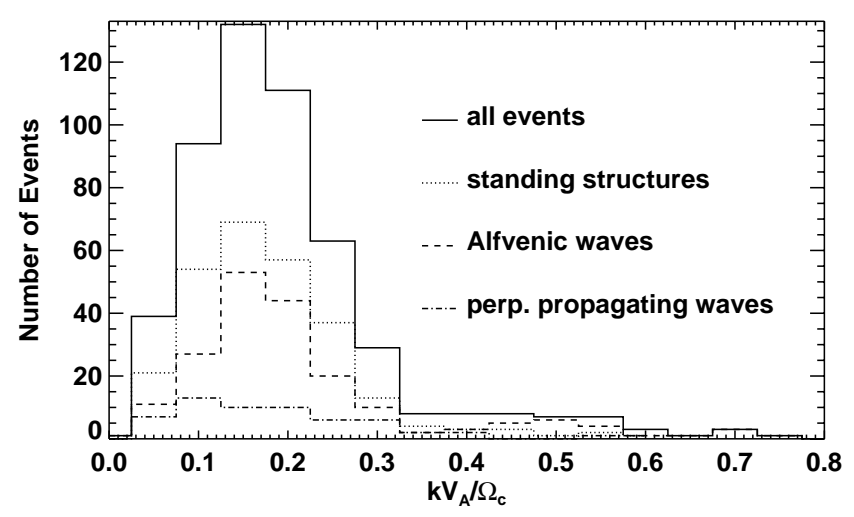

Fig. 13. Wave number histograms of all wave events and of different wave populations.

Alfvén modes. Since the slow mode is believed to be heavily damped under nominal magnetosheath plasma conditions (Krauss-Varban et al., 1994), one can assume that the discussed wave events belong to the class of Alfvénic waves. In other words, the accuracy of the determined phase velocities is good enough to identify wave events as Alfvénic waves, but Alfvén modes and slow modes cannot be distinguished. On the other hand, Stasiewicz (2005) suggest describing magnetosheath waves in a fluid picture, where it is not necessary to distinguish between both wave modes.

As a further method to organize the wave events identified, we use their wave numbers. For the total distribution, as well as the different wave populations previously identified we generate histograms of wave numbers $k$, normalized by $V_{A} / \Omega_{c}$ (Fig. 13). The distributions of the Alfvénic population and the standing structures show similarities concerning maximum and shape. In both cases we identified the largest number of waves around $k=0.15 \times \Omega_{c} / V_{A}$ with widths of the distributions between $k=0.05 \times \Omega_{c} / V_{A}$ and $k=0.3 \times \Omega_{c} / V_{A}$. The most frequent wave number of the 


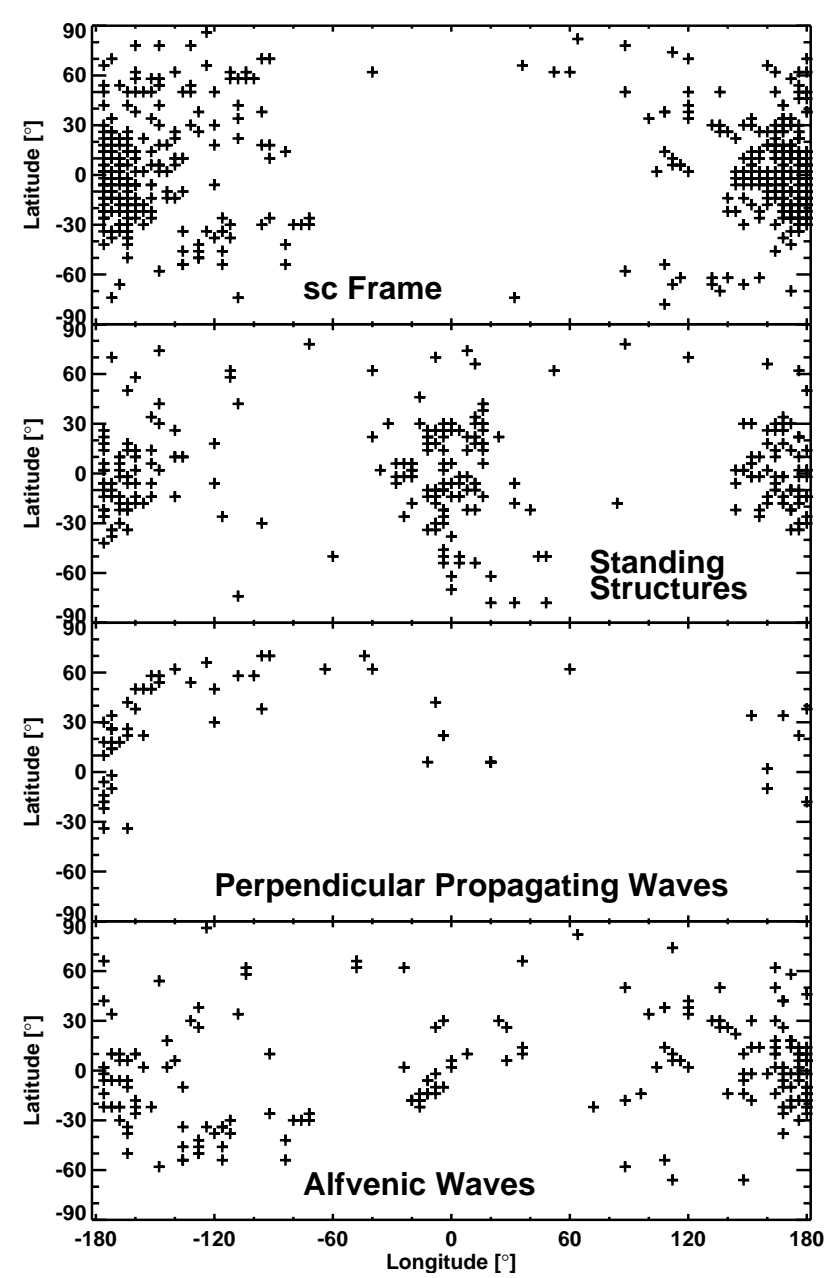

Fig. 14. Spatial distribution of the wave vector directions in the spacecraft frame (top), of standing structures (second panel), of perpendicular propagating wave (third panel) and of Alfvénic wave events (bottom) in the plasma rest frame.

perpendicular propagating wave events is at $k=0.1 \times \Omega_{c} / V_{A}$, buttheir total number of wave numbers is much smaller, which questions any comparison with the other two wave populations.

The magnitude of the non-normalized wave numbers is between $k=0.001 \mathrm{~km}^{-1}$ and $k=0.01 \mathrm{~km}^{-1}$. The configurations of the CLUSTER satellites are sensitive to wave numbers up to $k=0.032 \mathrm{~km}^{-1}$, so that our analysis is not restricted by spatial aliasing, due to the spacecraft separation. For the Alfvénic population and the standing structures, nearly $80 \%$ of the wave numbers are between $k=0.0015 \mathrm{~km}^{-1}$ and $k=0.0085 \mathrm{~km}^{-1}$, corresponding to wavelengths between $\lambda=740 \mathrm{~km}$ and $\lambda=4200 \mathrm{~km}$. Using a program presented by Gary (1992) the wave number of maximum growth rate of the proton-cyclotron instability and the mirror instability is found to be approximately $k=0.1025 \times \Omega_{c} / V_{A}$ for the conditions $\beta_{\|}=8.0, T_{\perp} / T_{\|}=1.5$ and $V_{A} / c=1 \times 10^{-4}$, where $c$ is the speed of light. This wave number is close to the maximum of the distribution in Fig. 13. But one has to keep in mind, that our analysis includes a large number of time intervals covering very different plasma properties, for example, plasma $\beta$ and the temperature anisotropy $T_{\perp} / T_{\|}$vary between each interval analyzed.

A wave propagation direction analysis with respect to the GSE coordinate system indicates further clear differences between the different wave populations found. In the spacecraft frame of reference wave vectors are mainly aligned in the direction $\left(\phi_{\text {lon }}= \pm 180^{\circ}, \phi_{\text {lat }}=0^{\circ}\right)$ (Fig. 14 , top), i.e. almost every wave event has a wave vector with a large negative $k_{x, G S E}$ component. This is in accord with our previous finding of the magnetosheath flow around the magnetopause organizing the wave propagation in the spacecraft frame of reference.

The spatial distribution of wave propagation directions in the plasma rest frame depends on the topology of the magnetic field lines within the magnetosheath. Field lines are draped around the magnetopause in such a way that they are almost aligned along this boundary layer (Spreiter et al., 1966; Kobel and Flückiger, 1994). Thus, waves propagating parallel to $\boldsymbol{B}$ should move along the magnetopause. Because the $k_{y}$ component is smaller than the $k_{x}$ component for most of the detected wave events (the y-axis corresponds to $\phi= \pm 90^{\circ}$ in the spherical representation, see Fig. 14), those wave events with $\theta_{k B} \approx 90^{\circ}$ should be directed towards the bow shock or the magnetosheath. For this reason the wave vectors of the standing structures are parallel or anti-parallel to the $X_{G S E}$-axis (Fig. 14, second panel). Wave vectors directed parallel to $X_{G S E}$, i.e. $\phi_{\text {lon }}=0^{\circ}, \phi_{\text {lat }}=0^{\circ}$, belong to wave events with negative phase velocities. In some cases the wave vectors of perpendicular propagating waves tend to be aligned with the magnetopause (Fig. 14, third panel).

As seen in the Friedrichs diagram (Fig. 12) the Alfvénic wave population exhibits a broad distribution of $\theta_{k B}$. In contrast to the standing structures and perpendicular propagating waves, the Alfvénic wave events have no favored propagation angle. Hence, the wave vectors of the Alfvénic population are scattered around the local magnetic field vectors and do not exhibit any preferred direction with respect to the GSE coordinate system (Fig. 14, bottom).

Figure 15 displays phase velocity vectors $\boldsymbol{V}_{p h}$ of the Alfvénic wave events in the GSE coordinate system. As expected from the Friedrichs diagram (Fig. 12) wave events with $V_{p h} \approx V_{A}$ propagate along the magnetopause, because of their quasi-parallel propagation angle $\theta_{k B}$. In contrast to this, slow velocity wave events of the Alfvénic wave population are directed towards the bow shock or magnetopause.

To compare wave vectors found with a classical analysis technique, we determine the propagation direction with the minimum variance analysis (MVA). The minimum variance direction $\boldsymbol{e}_{\min }$ is determined by the method of McPherron et al. (1972), using the real part of the spectral density matrix, so that $\boldsymbol{e}_{\min }$ can be estimated for a given frequency. Figure 16 shows the angle $\theta_{M V A}$ between $\boldsymbol{e}_{\min }$ and $\boldsymbol{B}$ plotted over $\theta_{k B}$. For quasi-perpendicular angles $\theta_{k B}$ the MVA leads to uniformly distributed values of $\theta_{M V A}$ between $0^{\circ}$ and $90^{\circ}$. One has to take into account that $\boldsymbol{e}_{\text {min }}$ does not correspond to 
the wave propagation direction for linear polarized waves, for example, mirror modes. Thus, only events with $\theta_{k B}<60^{\circ}$ are comparable with the means of MVA. In most cases the MVA delivers larger propagation angles than the wave telescope, while some angles $\theta_{M V A}$ are similar to the corresponding $\theta_{k B}$. The propagation direction of the wave telescope analysis can differ to $\boldsymbol{e}_{\min }$. However, the good correlation of the theoretical Friedrichs diagram and the phase velocity distributions of the Alfvénic wave events (Fig. 12) justifies the application of the wave telescope technique.

\section{Summary and conclusions}

The wave telescope method, as described in Sect. 3, was applied to magnetic field data of the CLUSTER satellites, and wave numbers and propagation directions have been evaluated unambiguously in the dayside magnetosheath. With additional information about plasma flow velocity and proton density information we have developed a survey of dispersion distributions, phase velocities, propagation angles $\theta_{k B}$ and propagation directions in the magnetosheath of fluctuations in the plasma rest frame.

The investigation of $(\omega, k)$-distributions and Friedrichs diagrams leads to three different populations of wave events. Most of the wave events are standing structures in the plasma rest frame, i.e. most wave events are convected structures in the magnetosheath plasma flow. These standing structures have the largest variation in the direction quasi-perpendicular to the magnetic field. In general, the magnetic field lines of the dayside magnetosheath are draped along the shape of the magnetopause. Thus, the standing structures are oriented towards the magnetopause and in the opposite direction towards the bow shock.

A small wave population featuring quasi-perpendicular propagation angles contains wave events with finite phase velocities below the Alfvén velocity. As these events exhibit an out-of-phase relationship when comparing density and magnetic field fluctuations, we tend to interpret them as mirror mode waves in a non-uniform magnetosheath, in which case we expect non-zero frequency, propagating global mirror mode structures.

As a third major population we detected a large number of wave events complying with the Alfvén branch of the Friedrichs diagram $V_{p h}=V_{A} \times \cos \theta$. The total number of wave events of the standing structures is slightly higher than the number of Alfvénic wave events. This result can be explained, because the selected time intervals are located closer to the magnetopause, where plasma conditions lead to a preferred growth of mirror modes. The decision as to which wave mode is represented by a wave event requires further in detailed case studies, especially for the perpendicular propagating wave events. Nevertheless, the wave telescope analysis confirms the generally accepted fact that mirror modes and Alfvén/proton-cyclotron waves are the predominant wave modes in the magnetosheath (e.g. Denton, 2000).

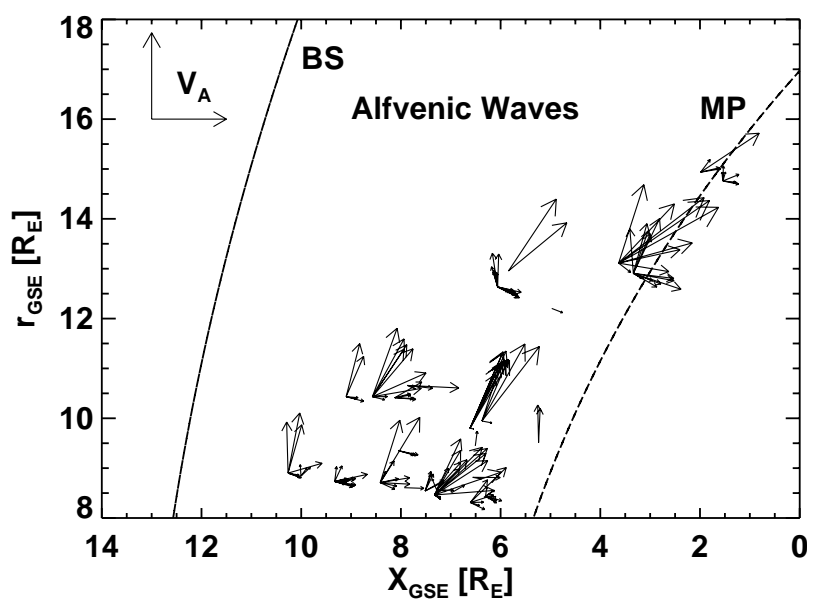

Fig. 15. Velocity vectors $V_{P h \text {,rest }}$ normalized to $V_{A}$ of the Alfvénic wave events in the GSE coordinate system with $r_{G S E}=\sqrt{Y_{G S E}^{2}+Z_{G S E}^{2}}$.

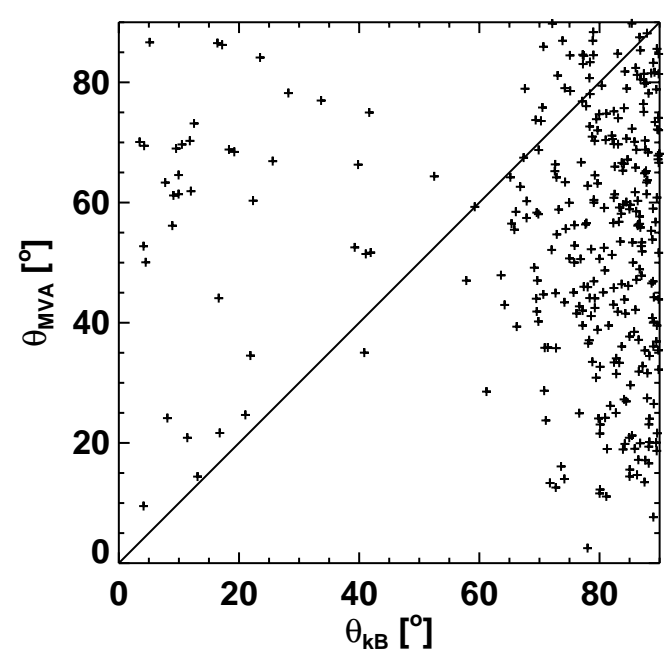

Fig. 16. Angle $\theta_{M V A}$ between $\boldsymbol{B}$ and the minimum variance direction $\boldsymbol{e}_{\min }$ compared to the propagation angle $\theta_{k B}$, determined by the wave telescope.

While the wave propagation directions differ between the three populations identified, the wave numbers and wave lengths are comparable. Typical wave lengths are of the order of 740-4200 km. If we assume a typical distance between the bow shock and the magnetopause, of the order of $20000 \mathrm{~km}$, this means that the majority of wave events are indeed propagating in the sheath and are not influenced by the boundaries of the magnetosheath. Only for the group of slowly quasi-perpendicular propagating global mirror modes do the spatial structures need to be considered. Future work will concentrate on this area. Also, while here we concentrated on magnetic field observations mainly, further studies are necessary and planned considering additional plasma parameters or various transport ratio parameters (Song et al., 1994; Denton et al., 1995). In particular, the implementation 
of wave mode filters (Glassmeier et al., 1995), together with the wave telescope algorithm, will enhance the analysis tool, in order to identify individual wave modes under different plasma conditions in the magnetosheath plasma environment. Furthermore, future statistical studies will reveal the differences between waves downstream of the quasi-parallel and the quasi-perpendicular bow shock, and will investigate the evolution of waves from the outer to the middle and inner magnetosheath. Also, the relation to waves upstream of the bow shock will be analyzed to expose the origin of magnetosheath waves.

Acknowledgements. The TU Braunschweig group was financially supported by the German Ministerium für Bildung und Forschung and the Deutsches Zentrum für Luft- und Raumfahrt under contract 50OC0103. The authors are also grateful to one of the referees for evaluating wave numbers of the proton-cyclotron instability using the code of Gary (1992).

Topical Editor T. Pulkkinen thanks two referees for their help in evaluating this paper.

\section{References}

Anderson, B. J., Fuselier, S. A., Gary, S. P., and Denton, R. E.: Magnetic spectral signatures in the Earth's magnetosheath and plasma depletion layer, J. Geophys. Res., 99, 5877-5886, 1994.

Balikhin, M. A., Schwartz, S., Walker, S. N., Alleyne, H. S. C. K., Dunlop, M., and Lühr, H.: Dual-spacecraft observations of standing waves in the magnetosheath, J. Geophys. Res., 106, 25 395-25 408, 2001.

Balogh, A., Carr, C. M., Acuna, M. H., and Dunlop, M. W.: The Cluster magnetic field investigation: overwiew of in-flight perfomance and initial results, Ann. Geophys., 19, 1207-1217, 2001, SRef-ID: 1432-0576/ag/2001-19-1207.

Chisham, G., Schwartz, S. J., Balikhin, M. A., and Dunlop, M. W.: AMPTE observations of mirror mode waves in the magnetosheath: Wavevector determination, J. Geophys. Res., 104, 437447, 1999.

Crooker, N. U. and Siscoe, G. L.: A mechanism for pressure anisotropy and mirror instability in the dayside magnetosheath, J. Geophys. Res., 82, 185-186, 1977.

Denton, R. E.: ULF waves in the magnetosheath, Intern. J. Geomagn. Aeronomy, 2, 1-14, 2000.

Denton, R. E., Gary, S. P., Li, X., Anderson, B. J., LaBelle, J. W., and Lessard, M.: Low-frequency fluctuations in the magnetosheath near the magnetopause, J. Geophys. Res., 100, 56655679, 1995.

Gary, S. P.: The mirror and ion cyclotron anisotropy instabilities, J. Geophys. Res., 97, 8519-8529, 1992.

Gedalin, M., Balikhin, M., Strangeway, R. J., and Russell, C. T.: Long-wavelength mirror modes in multispecies plasmas with arbitrary distributions, J. Geophys. Res. SP., 107(A2), SSH1, 1-6, 2002.

Glassmeier, K.-H., Motschmann, U., Dunlop, M., Balogh, A., Carr, C., Musmann, G., Fornacon, K.-H., Schweda, K., Vogt, J., Georgescu, E., and Buchert, S.: CLUSTER as a wave telescope first results from the fuxgate magnetometer, Ann. Geophys., 19, 1-10, 2001, Correction, Ann. Geophys., 21, 1071, 2003.
Glassmeier, K.-H., Motschmann, U., and v. Stein, R.: Mode recognition of MHD wave fields at incomplete dispersion measurements, Ann. Geophys., 13, 76-83, 1995.

Hasegawa, A.: Drift mirror instability in the magnetosphere, Phys. Fluids, 12, 2642-2650, 1969.

Hubert, D., Lacombe, C., Harvey, C. C., and Moncuquet, M.: Nature, properties, and origin of low-frequency waves from an oblique shock to the inner magnetosheath, J. Geophys. Res., 103, 26783-26798, 1998.

Johnson, J. R. and Cheng, C. Z.: Global structure of mirror modes in the magnetosheath, J. Geophys. Res., 102, 7179-7190, 1997.

Kobel, S. and Flückiger, E. O.: A model of the steady state magnetic field in the magnetosheath, J. Geophys. Res., 99, 23 617-23622, 1994.

Krauss-Varban, D., Omidi, N., and Quest, K. B.: Mode properties of low-frequency waves: Kinetic theory versus Hall-MHD, J. Geophys. Res., 99, 5987-6009, 1994.

Lacombe, C., Pantellini, F. G. E., Hubert, D., Harvey, C. C., Mangeney, A., Belmont, G., and Russell, C. T.: Mirror and Alfvenic waves observed by ISEE 1-2 during crossings of the earth's bow shock, Ann. Geophys., 10, 772-784, 1992.

McPherron, R. L., Russell, C. T., and Coleman, P. J.: Fluctuating Magnetic Fields in the Magnetosphere. II: ULF Waves, Space Science Reviews, 13, 411-454, 1972.

Motschmann, U., Woodward, T. I., Glassmeier, K.-H., and Dunlop, M. W.: Array Signal Processing Techniques, Proc. CLUSTER Workshop on Data Analysis Tools, ESA, SP-372, 104, 79-86, 1995.

Motschmann, U., Woodward, T. I., Glassmeier, K.-H., Southwood, D. J., and Pincon, J. L.: Wavelength and direction filtering by magnetic measurements at satellite arrays: Generalized minimum variance analysis, J. Geophys. Res., 101, 4961-4965, 1996.

Narita, Y., Glassmeier, K.-H., Schäfer, S., Motschmann, U., Fränz, M., Dandouras, I., Fornacon, K.-H., Georgescu, E., Korth, A., Rème, H., and Richter, I.: Alfvén waves in the foreshock propagating upstream in the plasma rest frame: Statistics from CLUSTER observations., Ann. Geophys., 22, 2315-2323, 2004,

SRef-ID: 1432-0576/ag/2004-22-2315.

Neubauer, F. M. and Glassmeier, K.-H.: Use of an Array of Satellites as a Wave Telescope, J. Geophys. Res., 95, 19 115-19 122, 1990.

Pinçon, J. L. and Lefeuvre, F.: Local characterization of homogenuous turbulence in a space plasma from simultaneous measurement of field components at several points in space, J. Geophys. Res., 96, 1789-1802, 1991.

Pokhotelov, O. A., Balikhin, M. A., Treumann, R. A., and Pavlenko, V. P.: Drift mirror instability revisited: 1 . Cold electron temperature limit, J. Geophys. Res., 8455-8464, 2001.

Rème, H., Aoustin, C., Bosqued, J. M., and Dandouras, I.: First multispacecraft ion measurements in and near the Earth's magnetosphere with the identical Cluster Ion Spectrometry experiment, Ann. Geophys., 19, 1303-1354, 2001,

SRef-ID: 1432-0576/ag/2001-19-1303.

Sahraoui, F., Pinçon, J. L., Belmont, G., Rezeau, L., CornilleauWehrlin, N., Robert, P., Mellul, L., Bosqued, J. M., Balogh, A., Canu, P., and Chanteur, G.: ULF wave identification in the magnetosheath: The k-filtering technique applied to Cluster II data, Journal of Geophysical Research (Space Physics), 108(A9), SMP, 1-18, 2003, correction, J. Geophys. Res., 109(A4), SMP, 4222, 2004.

Sahraoui, F., Belmont, G., Pinçon, J. L., Rezeau, L., Balogh, A., Robert, P., and Cornilleau-Wehrlin, N.: Magnetic 
turbulent spectra in the magnetosheath: new insights, Ann. Geophys., 2283-2288, 2004.

Schwartz, S. J., Burgess, D., and Moses, J. J.: Low-frequency waves in the Earth's magnetosheath: Present status, Ann. Geophys., 14, 1134-1150, 1996,

\section{SRef-ID: 1432-0576/ag/1996-14-1134.}

Sckopke, N., Paschmann, G., Brinca, A. L., Carlson, C. W., and Lühr, H.: Ion thermalization in quasi-perpendicular shocks involving reflected ions, J. Geophys. Res., 95, 6337-6352, 1990.

Song, P., Russell, C. T., and Gary, S. P.: Identification of lowfrequency fluctuations in the terrestrial magnetosheath, J. Geophys. Res., 99, 6011-6025, 1994.
Spreiter, J. R., Summers, A. L., and Alksne, A. Y.: Hydrodynamic flow around the magnetosphere, Planetary Space Sci., 1, 223 253, 1966

Stasiewicz, K.: Reinterpretation of mirror modes as trains of slow magnetosonic solitons, Geophys. Res. Lett., 31, L21 1-5804, 2004a.

Stasiewicz, K.: Theory and Observations of Slow-Mode Solitons in Space Plasmas, Physical Review Letters, 93, 125 004, 1-4, 2004b.

Stasiewicz, K.: Nonlinear Alfvén, magnetosonic, sound, and electron inertial waves in fluid formalism, J. Geophys. Res. SP., 110(A03220), 1-9, 2005. 